\title{
High yield criteria for emergency cranial computed tomography in adult patients with no history of head injury
}

\author{
J E Harris, H L Draper, A I Rhodes, J M Stevens
}

\begin{abstract}
Objectives-A recent American study identified clinical factors which effectively predicted those patients who would have significant findings on cranial computed tomography. It was proposed to apply these criteria in a UK setting and to determine whether modifications could be made to improve their efficiency.

Methods-A prospective observational study was conducted over a four month period including all non-trauma adult patients referred from the accident and emergency ( $A \& E$ ) department for urgent cranial computed tomography. Presenting symptoms and signs were analysed for ability to predict clinically significant computed tomography findings, namely: acute infarct, malignancy, acute hydrocephalus, intracranial haemorrhage, or intracranial infection.

Results-Sixty two patients were included; $22(35 \%)$ had significant findings on computed tomography. Applying the original criteria (any of: age 60 years or older, focal neurology, headache with nausea or vomiting, altered mental status) to the study population showed that no clinically significant tomograms would have been omitted but only $11 \%$ fewer performed. Modifying the criteria by removing "age 60 years or older" and replacing "altered mental status" with a Glasgow coma score $<14$, still ensured $100 \%$ sensitivity and would have resulted in $19 \%$ fewer scans being performed.

Conclusion-Simple clinical criteria can be usefully applied to patients presenting to an A\&E department in this country to target patients most likely to have clinically significant findings on urgent cranial computed tomography.

(F Accid Emerg Med 2000;17:15-17)
\end{abstract}

Keywords: computed tomography; cranial; guidelines

Registrar in Accident and

Emergency, Accident and

Emergency Department, St

George's Hospital,

Blackshaw Road, London

SW17 0QT

Accepted 28 July 1999

Emergency cranial computed tomography is frequently requested for non-trauma patients presenting to the accident and emergency
(A\&E) department with new neurological symptoms and signs.

Emergency radiological procedures are essential but costly in financial terms and also in terms of time and manpower. A study analysing out of hours emergency radiological procedures concluded that although most procedures were justified, others including nontrauma cranial computed tomography could be performed less frequently. ${ }^{1}$

Well recognised guidelines are available for the use of cranial computed tomography in patients who have sustained a head injury, ${ }^{23}$ but currently no widely accepted guidelines exist for non-trauma patients.

Earlier studies were unable to derive useful criteria to identify patients most likely to have clinically significant cranial abnormalities on computed tomography. ${ }^{45}$ In these studies, trauma and non-trauma patients were evaluated as a single group. A more recent study by Rothrock and colleagues, which included only non-trauma patients, proved more successful in deriving criteria. ${ }^{6}$ They found that if cranial computed tomography was restricted to only patients fulfilling any of four clinical criteria (age 60 years or older, focal neurology, headache with nausea or vomiting, altered mental status), then no significant pathology would have been missed and $28 \%$ fewer scans would have been performed.

The purpose of our study was to investigate the application of these criteria to the patients referred for non-trauma, emergency cranial computed tomography from an A\&E department in the UK. We examined other clinical features to determine whether modifications could be made to improve the efficiency of these criteria without missing a single significant, positive scan.

\section{Method}

A prospective observational study was conducted over a four month period (February to May 1998). The study was conducted in a busy central London teaching hospital where the annual attendance at the $\mathrm{A} \& \mathrm{E}$ department is 69000. history of head injury, who were referred from the $\mathrm{A} \& \mathrm{E}$ department for an emergency cranial 
computed tomography, were included in the study. Patients were excluded when there was a strong suspicion of trauma, for example patients presenting with a reduced level of consciousness who had bruising to the face or scalp.

Relevant clinical data were collected each day from the A\&E notes, medical records, the computed tomography request form, and radiologist's report. All scans were reported by a consultant radiologist who was aware only of the clinical details supplied on the request form.

We considered the following findings on computed tomography to be clinically significant: acute infarct, malignancy, acute hydrocephalus, intracranial haemorrhage, and intracranial infection. All other findings were defined as not significant for the purpose of our study.

Presenting symptoms and signs were analysed for ability to predict clinically significant computed tomography findings.

STATISTICAL METHODS

The data were set up in $2 \times 2$ tables. The variables "criteria present" or "criteria absent" were tabulated against clinically significant or not significant computed tomography findings.

The sensitivity of the criteria was calculated with a one sided $97.5 \%$ confidence interval (CI), as the value of one of the cells in the table was zero. The sensitivity in this case signifies the proportion of the patients found to have significant computed tomography findings who were actually selected by the criteria.

The specificity indicates the ability of the criteria to exclude those patients who did not have clinically significant computed tomography findings. The specificity was calculated with a $95 \% \mathrm{CI}$.

\section{Results}

Sixty two patients were included; 22 (35\%) had clinically significant findings on computed tomography (table 1).

Applying the original criteria (any of: age 60 years or older, focal neurology, headache with nausea or vomiting, altered mental status) to

Table 1 Significant findings on computed tomography

\begin{tabular}{ll}
\hline Findings & $\begin{array}{l}\text { No of } \\
\text { patients }\end{array}$ \\
\hline Acute infarct & \\
$\quad$ Non-haemorrhagic & 5 \\
With secondary haemorrhage & 1 \\
Intracranial haemorrhage & \\
$\quad$ Subarachnoid & 4 \\
$\quad$ Intracerebral & 8 \\
Malignancy & 4 \\
\hline
\end{tabular}

Table 2 Results based on original criteria

\begin{tabular}{|c|c|c|c|}
\hline \multirow[b]{2}{*}{ Criteria } & & \multicolumn{2}{|l|}{ Findings } \\
\hline & & Significant & Non-significant \\
\hline $\begin{array}{l}\text { - Age } \geqslant 60 \text { years } \\
\text { Altered mental status } \\
\text { - Focal neurology } \\
\text { - Headache with } \\
\text { nausea or vomiting }\end{array}$ & $\begin{array}{l}\text { Present } \\
\text { Absent }\end{array}$ & $\begin{array}{r}22 \\
0\end{array}$ & $\begin{array}{r}33 \\
7\end{array}$ \\
\hline
\end{tabular}

Table 3 Results based on modified criteria

\begin{tabular}{|c|c|c|c|}
\hline \multirow[b]{2}{*}{ Criteria } & & \multicolumn{2}{|l|}{ Findings } \\
\hline & & Significant & Non-significant \\
\hline - Glasgow coma score $<14$ & Present & 22 & 29 \\
\hline $\begin{array}{l}\text { - Focal neurology } \\
\text { - Headache with nausea } \\
\text { or vomiting }\end{array}$ & Absent & 0 & 11 \\
\hline
\end{tabular}

our study population showed no clinically significant computed tomography findings would have been omitted and $11 \%$ fewer scans could have been performed (table 2). Statistical analysis showed $100 \%$ sensitivity $(97.5 \%$ CI $85 \%$ to $100 \%)$ and $18 \%$ specificity $(95 \%$ CI $7 \%$ to $33 \%$ ). The clinical features used in the original criteria are not efficient at excluding patients who have non-significant computed tomography findings.

Modifying the clinical criteria (any of: focal neurology, headache with nausea or vomiting, Glasgow coma score $<14$ ), we found that, still, no significant pathology would have been missed and there would have been an even greater reduction in the number of scans performed (19\%) (table 3). Statistical analysis showed $100 \%$ sensitivity $(97.5 \%$ CI $85 \%$ to $100 \%$ ) and $28 \%$ specificity (95\% CI $22 \%$ to $33 \%)$. Modification of the criteria improved patient selection for emergency cranial computed tomography.

\section{Discussion}

Cranial computed tomography is an important investigation, particularly in the emergency situation but it involves exposure to ionising radiation, and also moves a potentially unstable patient away from the A\&E department which may delay other more relevant investigations or treatment. The development of guidelines to assist in determining whether an emergency computed tomogram has a high or low likelihood of significant findings may, therefore, be helpful.

The authors of the previous study commented that the definition of "altered mental status" was subjective and we felt that it should be replaced by a more objective measurement. The Glasgow coma score is known to be reliable and reproducible, thus it would reduce interobserver variability. It is routinely recorded in patients attending the $\mathrm{A} \& \mathrm{E}$ department with neurological problems. We chose to use a score of $<14$ because, although analysis of our results revealed that using a score as low as 8 would have been equally sensitive, our study population was small and we were concerned that in a larger group some significant findings might have been missed.

Overall, the proportion of significant computed tomography findings in the 60 years or older group $(59 \%)$ was higher than in the younger patients $(23 \%)$. When a combination of clinical features are to be considered, the clinical condition of the patient is more important than the age in the decision to request a cranial scan. In our population, 23 patients were 60 years or older and all except two fulfilled other criteria - both these two patients 
had non-significant computed tomography findings. The removal of " 60 years or older" as a criterion enabled the specificity of the combined criteria to be increased while maintaining $100 \%$ sensitivity.

Other clinical features that were considered for inclusion in the criteria were a history of headache without nausea or vomiting and history of seizure. The inclusion of these features was not found to improve the efficiency of the combined criteria.

When the original criteria were applied to our patient population, we found a smaller potential reduction in the number of computed tomograms $(11 \%)$ than that reported by Rothrock et al (28\%) and a lower specificity $(18 \% v 31 \%)$. A higher proportion of our patients would have fulfilled the original criteria than those in the American study. The rate of abnormal computed tomography findings in our population was $35 \%$ (v $8 \%$ ), indicating that in this country clinicians were already selecting patients who had a higher likelihood of having an abnormal scan. This may explain the differences in the results.

Although our study is concerned with selecting patients likely to have clinically significant findings on cranial computed tomography, it is conceded that the finding of a negative scan can also be important. When a patient does not meet the suggested clinical criteria, the clinician may still feel that urgent computed tomography is appropriate as, for example, in the case of a "thunderclap" headache without nausea or vomiting, which may signify a subarachnoid haemorrhage. ${ }^{7}$

Before the results of this study could be recommended as guidelines for clinical practice they would need to be further validated in a larger population, so that less typical presentations could be included.

The criteria identified by Rothrock and colleagues have been successfully applied to the population attending an A\&E department in the UK. Although this was a small scale study, similar results were achieved. The suggested modifications are easy to administer and improve the specificity of the criteria. In conclusion, simple clinical criteria can be helpful to target patients most likely to have clinically significant findings on urgent cranial computed tomography.

Conflict of interest: none.

Funding: none.

Contributors

Julia Harris participated in the study design, data collection, and writing the paper. Helen Draper participated in the data documentation and analysis and contributed to the writing of the paper and prepared the manuscript. Anita Rhodes initiated the research, participated in the study design, data collection, statistical analysis, and contributed to the paper. John Stevens discussed core ideas and interpretation of findings, reported the computed tomograms, and edited the paper.

John Stevens is the guarantor for the paper.

1 Wong DC, Siddle KJ. After-hours radiology. Australian Radiology 1994;38:326-30.

2 Briggs M, Clarke P, Crockard A, et al. Guidelines for the initial management after head injury in adults. Suggestions from a group of neurosurgeons. BMF 1984;288:983-5.

3 Teasdale GM, Murray G, Anderson E, et al. Risks of acute traumatic intracranial haematoma in children and adults: mplications for managing head injuries. BMF 1990;300: 363-7.

4 Mills ML, Russo LS, Ross BA. High yield criteria for cranial computed tomography scans. Ann Emerg Med 1986;15: 1167-72.

5 Reinus WR, Zwemer FL. Clinical prediction of emergency cranial computed tomography results. Ann Emerg Med 1994;23:1271-8.

6 Rothrock SG, Buchanan C, Green SM, et al. Cranial computed tomography in the emergency evaluation of adult patients without a recent history of head trauma: a prospective analysis. Acad Emerg Med 1997;4:654-61.

7 Neil-Dwyer G, Lang D. "Brain attack"-aneurysmal Nel-Dwyer G, Lang D. "Brain attack"-aneurysmal
subarachnoid haemorrhage: death due to delayed diagnosis. f R Coll Physicians Lond 1997;31:49-52. 